arterial blood and consequently the oxygen content of mixed venous blood falls. Though arterial oxygen tension is often reduced in myocardial infarction (McKenzie et al., 1964) arterial oxygen content is reduced in only the most severely ill and is unlikely to cause a fall in mixed venous saturation. Within these limits mixed venous oxygen saturation is likely to be a good index of cardiac output.

There are difficulties in obtaining a true measurement of venous oxygen saturation. In some patients oxygen saturation will be too low to allow an adequate estimation by a haemoreflector technique (Cole and Hawkins, 1967). The measurement of oxygen tension and $\mathrm{pH}$ allow conversion to saturation, the data of Severinghaus (1966) being used. The conversion, however, will be made on the steep portion of the haemoglobin dissociation curve and small errors in measurement of oxygen tension could be magnified on conversion to saturation; thus a 1-mm. error in oxygen tension will lead to a $3 \%$ error in venous oxygen saturation. Moreover, possibly all patients do not have the same haemoglobin dissociations curves (Metcalfe et al., 1969). Despite these technical considerations the measurement of oxygen tension and conversion to saturation is adequate for clinical purposes. Ideally total oxygen content should be measured, but the sample volume required for the standard method is large and difficult to obtain through a float catheter. Micromethods for the measurement of total oxygen content are available and are likely to be improved (Laver, 1967; Linden et al., 1965).

Scheinman et al. (1969) showed that central venous oxygen saturations collected from atrial level correlated well with true mixed venous oxygen saturations in patients with uncomplicated myocardial infarction. In patients who are severely ill with shock or failure the correlation between saturations from the great veins and pulmonary artery is less close. This can be explained on the basis of differing regional circulation in patients with low output states. Thus a true mixed venous blood sample will provide a better guide for the monitoring of patients with shock or left ventricular failure. Pulmonary arterial catheterization by the float technique is relatively simple and safe. The measurement of mixed venous oxygen saturation will allow a rational approach to the treatment of patients with shock or failure complicating acute myocardial infarction with the use of the minimum of equipment.

We thank Professor K. W. Donald for his help and encouragement, the members of the medical and nursing staff who aided in these studies, and Mr. J. Walker and Mrs. C. Simpson for technical help.

\section{REFERENCES}

Cole, P. V., and Hawkins, L. H. (1967). Bio-Medical Engineering, 2, 56 Flenley, D. C., Millar, J. S., and Rees, H. A. (1967). British Medical Fournal, 2, 349.

Goldman, R. H., Braniff, B., Harrison, D. C., and Spivack, A. P. (1968). Annals of Internal Medicine, 68, 1280.

Laver, M. B. (1967). Fournal of Applied Physiology, 22, 1017

Linden, R. J., Ledsome, J. R., and Norman, J. (1965). British fournal of Anaesthesia, 37, 77.

MacDonald, H. R., et al. (1967). Lancet, 1, 1070

McKenzie, G. J., et al. (1964). Lancet, 2, 825.

Metcalfe, J., Dhindsa, D. S., Edwards, M. J., and Mourdjinis, A. (1969). Circulation Research, 25, 47.

Oriol, A., McGregor, M. (1967). American fournal of Cardiology, 20, 826. Scheinman, M. M., Brown, M. A., and Rapaport, E. (1969). Circulation, $40,165$.

Severinghaus, J. W. (1966). Fournal of Applied Physiology, 21, 1108.

Shubin, H., Afifi, A. A., Rand, W. M., and Weil, M. H. (1968). Cardiovascular Research, 2, 329.

Valentine, P. A., et al. (1966). Lancet, 2, 837.

Van Slyke, D. D., and Neill, J. M. (1924). Fournal of Biological Chemistry, $61,523$.

World Health Organization (1959). Technical Report Series, No. 168. Zimmerman, H. A. (1966). Intravascular Catheterization, 2nd. edn.

\title{
Choreoathetotic Movement Disorder in Alcoholism
}

\author{
P. J. MULLIN, ${ }^{*}$ M.B., B.S., D.P.M. ; P. W. KERSHAW, $†$ M.R.C.P.ED., D.P.M., D.oBST.R.C.o.G.
}

J. M. W. BOLT, $\ddagger$ M.B., B.SC., D.P.M.

British Medical fournal, 1970, 4, 278-281

\begin{abstract}
Cummary: A syndrome of choreoathetosis in association with alcoholism has been found in 12 patients. It appeared to occur more often in women, was transient, and may have been associated with alcohol withdrawal. It was not associated with gross liver disease, phenothiazine administration, or familial chorea, and no consistent abnormalities in whole blood thiamine or nicotinic acid, in serum magnesium, or in serum vitamin $B_{12}$ levels were present.
\end{abstract}

\section{Introduction}

While neurological complications of alcoholism have been described for over a hundred years, choreoathetosis has only been indirectly mentioned in association with alcoholism, in so far as it occurs as a complication of advanced hepatic cirrhosis, usually with a portosystemic shunt (Victor et al., 1965) or as a manifestation of hypomagnesaemia (MacIntyre, 1963).

\footnotetext{
* Consultant Psychiatrist, Southern General Hospital, Glasgow S.W.1.

† Consultant Psychiatrist, Ravenscraig Hospital, Greenock, Renfrewshire. ‡ Consultant Psychiatrist, Central Hospital, Warwick. Late Senior Registrar, Western Regional Hospital Board, Scotland.
}

\section{Patients and Methods}

Twelve patients (six women and six men) were admitted to hospital on account of alcoholism or its complications between December 1967 and July 1969. Though their age range was from 29 to 67 years, the mean age of 48 was about five years greater than that of alcoholics admitted to one of the units. Half the patients drank mainly whisky, four mainly wine, one beer, and one sherry and cider. This is typical of Glasgow alcoholics, where beer is usually taken as an adjuvant to more potent drinks.

Family histories were taken by one of us (J. B.) who has had considerable experience in obtaining these in connexion with work on Huntington's chorea (Bolt, 1970). All investigations were done as soon as possible after admission. Wholeblood thiamine and nicotinic acid were measured microbiologically, using modifications of the original methods of Edwards et al. (1957) and Sarett et al. (1946), respectively. Serum folate was measured by the method of Waters and Mollin (1961) and serum vitamin $B_{12}$ by that of Hutner et al. (1956). The serum copper oxidase was measured by the method of Ravin (1961) and the serum magnesium by an atomic absorption method. 


\section{Results}

The abnormal movements were commonly present in the upper half of the body and were essentially transient in character. There was a wide variety of facial movements, some patients showing eyebrow raising or blinking, others tongue protrusion. Almost all had grimacing of the perioral muscles. The facial movements when present were characteristically rapid, and laterality was shown by four cases. The oral movements had a typically twisting, pursing character; the hand and forearm movements had a sinuous, writhing character while the leg movements tended to be sudden and jerky. In 10 of the 12 patients abnormal movements were present on admission; in the other two they appeared on the fourth and fifth days after admission. Though the movements are described as "transient," their duration varied from a few hours in Case 8 to a year in Case 1. One patient (Case 9) still had reduced abnormal movements five months after they were first noted, though the possibility that he continued to drink cannot be entirely excluded. Nevertheless, there appears to be no recurrence of the movements if the patient remains abstinent. One patient (Case 4) had a return of abnormal movements when he resumed drinking as an inpatient. In two patients (Cases 3. and 10), who have been known over a period of years, the clinical picture has fluctuated, the movements being much more obvious during periods of drinking and gradually diminishing during periods of abstinence.

The only other neurological abnormalities found were muscle weakness in Cases 1, 2, 3, 4, and 6; sensory impairment in Cases 1, 3, and 4; and finger-nose dysmetria in Case 2 , this being the only sign of cerebellar dysfunction in the series. Combaratively minor degrees of hepatomegaly were found in Cases 4, 9, and 3, the latter showing the only other clinical sign of hepatic impairment in the series-that is, prominent vascular spiders on the legs.

Routine tests of liver function showed only minor abnormalities-the serum transaminase levels are shown in the Table; the serum bilirubin and zinc sulphate turbidity levels were slightly raised in Cases 12 and 5, respectively, alpha 2 -globulins were increased in Cases 1 and 6 and gammaglobulin in Case 3. The bromsulphthalein retention was increased in Cases 1 and 7 (17\% and 14\%, respectively), falling to normal one month after admission. Whole-blood thiamine and serum folate estimations are shown in the Table; serum $B_{12}$, nicotinic acid, and magnesium were normal in all patients in whom they were estimated. Serum copper oxidase, calcium, phosphàte, and uric acid were normal in all cases. No patient had an abnormal E.E.G. One (Case 4) had a slight degree of pneumonic consolidation on admission and another (Case 6), who was aged 67, showed E.C.G. evidence of ischaemia, otherwise no other abnormalities were found. Liver biopsies are reported in the case histories.

Full psychological testing was carried out in eight patients: three (Cases 7, 8, and 10) showed no sign of intellectual impairment; four (Cases 3, 4, 9, and 11) showed slight changes, including memory impairment, general deterioration on performance tests, perseveration, and confabulation; and one (Case 1) showed a more pronounced impairment, and though her tests improved during her stay of 18 months in hospital she still had amnesia for a period of some months about the time of her index admission.

\section{Illustrative Case Histories}

Case 1.-A woman born in 1916 started to take excess alcohol in 1963 and was first admitted to hospital in August 1967. She gave a history of taking a "bottle of wine" daily for eight months. She settled well with minimal withdrawal symptoms but failed to attend for follow-up. In January 1968 she was readmitted to a medical ward with complaints of weakness, limb pain, and vomiting. She was emaciated and had typical choreiform movements. Severe peripheral neuropathy, ophthalmoplegia, and a variable confusional state were also present. A diagnosis of Wernicke's encephalopathy was made. Six hours after administration of intravenous Parentrovite she was more alert, replied more rapidly to questions, and was able to move her limbs on command. During the next few days she developed pronounced withdrawal symptoms and subsequently a Korsakoff psychosis, which persisted for three months. Her choreiform movement disorder and peripheral neuropathy gradually improved so that she was walking with aid nine months after admission and walked unassisted on discharge at 18 months. Liver biopsy some months after admission showed no abnormality. She had no history of head injury, phenothiazine intake, or family history of Huntington's chorea; her mother and brother drink heavily.

Case 2.-A woman born in 1926 was admitted to hospital in 1969 with a complaint of leg weakness of three months' duration and anorexia for the preceding month. During the year before admission she had taken a "quarter to half" a bottle of whisky daily. On examination she had profound weakness of the muscles of the face and upper and lower limbs. Choreiform movements were present in the hands and forearms, being more pronounced on the right than the left side. Tendon reflexes were absent in the lower limbs, and the plantar responses were flexor. She was disorientated and confabulating. After a period of initial improvement her condition suddenly deteriorated and she died of bronchopneumonia a week after admission. She had never received phenothiazines. There was no family history of alcoholism or neurological disease. At necropsy the liver weighed 1,800 g., histological examination showing fatty change with bile duct proliferation and minimal fibrosis. The brain (examined by Dr. J. Hume Adams) weighed 1,300 g., no abnormalities being noted on macroscopic examination. Histological examination, however, revealed the typical changes of a rather subacute Wernicke's encephalopathy. In addition, some rarefaction and reactive gliosis in the wall of the third ventricle and a very light fibrillary gliosis here and there in the white matter were found. There was no evidence of degenerative changes in the anterior thalamic nuclei or basal ganglia.

Case 3.-A woman born in 1913 started to take excess alcohol in 1962, probably half a bottle of whisky daily. She was first referred in 1965 and since then has had many admissions both to psychiatric and to medical wards on account of alcoholism or its complications-for example, visual hallucinations, self-poisoning attempts, septicaemia, and occasional epileptic seizures. She developed choreoathetotic movements in 1967. The movements gradually ceased after admission, alcohol being then withdrawn and, as a rule, parenteral vitamins being given. On one welldocumented occasion, however, parenteral vitamins were withheld and the abnormal movements persisted until these were given one month later. Liver biopsy in 1968 showed a slight degree of fatty change and some evidence of cirrhosis without necrosis. Her father died at the age of 52 years from "alcoholic" liver cirrhosis. Her brother had haemochromatosis and cerebellar signs. In the past she had received chlorpromazine but never in excessive dosage.

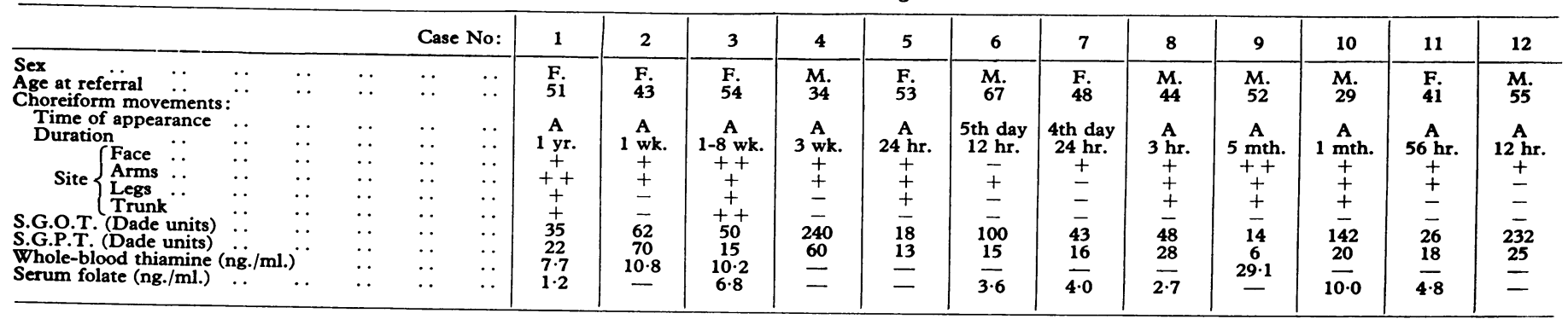

$\mathbf{A}=$ Present on admission. $\quad+=$ Present. $\quad-=$ Absent. 


\section{Discussion}

The syndrome described here is thus a transient choreoathetosis more usually affecting the upper half of the body. The picture is similar to that described by Hunter et al. (1964) secondary to prolonged administration of phenothiazine drugs. There was, however, no evidence that any of these patients had received phenothiazine drugs over prolonged periods or even in high doses over short periods. Chlorpromazine, in fact, had a beneficial effect on the movements of one patient (Case 10). Another (Case 9) was known to have taken excessive quantities of diazepam before admission, but there is no reason to suspect this drug as a possible aetiological factor.

Women appear more prone to develop choreiform movements with both phenothiazines and alcohol; the ratio of men to women is about $4: 1$ in the total admissions for alcoholism to one of our psychiatric units over a period of four years. The sex ratio of $1: 1$ in the cases described here thus shows a preponderance of females.

Possibly these represent early cases of Huntington's chorea, but detailed family histories have shown no evidence of any neurological disorder apart from that referred to in Case 3. While denial of the family history is not uncommon in Huntington's chorea for various reasons (Hans and Gilmore, 1968), no connexion between these families and any of the families with Huntington's chorea in the western region of Scotland previously collected by Bolt (1970) was established. In the latter study excessive drinking was alleged to have occurred in 24 men and four women from the total of 695 patients for whom records were available or who were reliably reported as suffering or having suffered from Huntington's chorea. The sex ratio of $6: 1$ in the patients with Huntington's chorea thus resembles that in uncomplicated alcoholism rather than that of the cases reported here. Other heredofamilial degenerations such as Wilson's disease are similarly excluded by the absence of a family history, gross liver disease, and the characteristic neurological, biochemical, and radiological findings.

Choreiform movements have been observed previously in the presence of severe liver disease and portosystemic shunts (Victor et al., 1965; Hurwitz and Montgomery, 1965; Toghill et al., 1967). Victor et al. (1965) studied 27 patients with severe liver disease (23 of whom had previous portal encephalopathy) who developed a chronic neurological disorder which they called hepatocerebral degeneration. Of these 27, 11 were alcoholics and some of these exhibited typical choreoathetotic movements: one was said to be indistinguishable from Huntington's chorea. They seem to have had more severe liver disease than the patients we have described. While liver function tests were completely normal in only two of our 12 patients the changes in the remaining ten were minor and similar to those normally found in alcoholics admitted to the units. Thus none of our patients, including the two with histological evidence of liver damage, has ever had ascites or oedema though three (Cases 2, 3, and 9) gave a history of previous "hepatitis." Possibly Case 3 could be suffering from chronic hepatocerebral degeneration although she has never had any episode of portosystemic encephalopathy and in periods of abstinence, providing that she is well nourished, the choreiform movements disappear. Victor et al. (1965) did not describe complete remission of the movements in their paper. Thus possibly all our patients are suffering from a very early form of chronic hepatocerebral degeneration, especially as the movements we describe are so transient.

Nutritional factors must always be considered in any condition which is related to alcoholism. Owing to the frequency with which oral or parenteral vitamins are administered to alcoholics useful estimations of blood thiamine and nicotinic acid levels could be obtained in only a few cases; of these the blood thiamine was low in three (Cases 1, 2, and 3) whereas the nicotinic acid levels were within normal limits. The fact that on admission two of the patients (Cases 1 and 2) were suffering from Wernicke's encephalopathy, a very rare condition, in itself suggests a causal relationship. The diet of five other patients was poor but that of the remainder adequate. In general patients who had had a poor diet had more persistent movements, and, as noted earlier, on one occasion one patient had a rapid improvement in her movements following treatment with parenteral vitamins a month after admission. There is, however, no evidence from the literature that patients with Wernicke's encephalopathy exhibit choreoathetosis. Jolliffe et al. (1940) described a severe encephalopathy with cog-wheel rigidity responding to nicotinic acid but not to thiamine and occurring in patients on a poor diet, some of whom had classical pellagra.

Despite the lack of definitive support for a deficiency of thiamine or nicotinic acid in our findings, Woods and Pendleton (1925) reported choreoathetosis in association with dietary deficiency states, and the latter must remain one of the more likely causes. Interestingly Alexander (1942), in describing an alcoholic with severe cirrhosis who developed rhythmic tremors of the hands, arms, mouth, and eyes-in other words the condition which would now be called acquired hepatocerebral degeneration-postulated that the aetiology might be "lack of a metabolic catalyst or vitamin."

Hypomagnesaemia was not found in those patients in whom the serum magnesium was estimated. Though choreiform movements are described in relation to hypomagnesaemia it is not clear whether they are related to magnesium depletion, coincidental liver disease (Fishman, 1965), or to some of the other deficiencies mentioned in many of these reports-for example Flink et al. (1954) and Hammarsten and Smith (1957). Since magnesium diuresis does occur in alcoholics (Kalbfleisch et al., 1963) minor fluctuations in its level might possibly interfere with the functioning of enzyme systems within the hepatic cell.

The transient nature of the disorder suggests that it may be related to alcohol withdrawal; the relationship was quite definite in Cases 5, 6,7 , and 8, but Cases 3, 7, and 10 showed the movements while drinking heavily. There was no apparent correlation between the extent of distribution of the condition and the type of beverage consumed or the duration of alcoholism. It is not clear why the condition is more common in female alcoholics.

The neuropsychiatric syndromes associated with liver disease have generally been attributed to failure of the liver to detoxify protein breakdown products. Victor et al. (1965) suggested that a subtle abnormality of liver function may exist in those cases in which neurological disorder antedates detectable derangement of liver function or ammonium metabolism. Such an abnormality could presumably result from a disruption of intracellular systems secondary to a deficiency of a variety of factors, known or unknown.

The only constant aetiological factor in our cases is their high alcohol intake. It seems not unreasonable, however, to suppose that they represent an early transient form of the chronic hepatocellular degeneration of Victer et al. (1965). This, together with the existence of Wernicke's encephalopathy in two of our cases and the probability or possibility of vitamin deficiency in several more (poor diet, gastritis, motor, sensory, or memory impairment, the latter being noted in all but two cases), opens up an interesting field for speculation.

We are grateful to Professor T. Ferguson Rodger for his encouragement in the preparation of this paper and to the various consultants who were kind enough to refer patients. We are indebted to Dr. G. Crean for performing liver biopsies, to Dr. J. Adams for serum $B_{12}$ estimations, to Dr. J. Hume Adams for his study of the brain of Case 2, and to Dr. A. Fell for serum magnesium estimations. 
Alexander, L. (1942). Research Publications, Association for Research in Nervous and Mental Disease, 21, 334.

Bolt, J. M. W. (1970). British fournal of Psychiatry, 116, 259.

Edwards, M. A., Kaufman, M. L., and Storvick, C. A. (1957). American fournal of Clinical Nutrition, 5, 51.

Fishman, R. A. (1965). Archives of Neurology, 12, 562.

Flink, E. B., Stutzman, F. L., Anderson, A. R., Konig, T., and Fraser, R. (1954). Fournal of Laboratory and Clinical Medicine, 43, 169.

Hammarsten, J. F., and Smith, W. O. (1957). New England fournal of Medicine, 256, 897 .

Hans, M. B., and Gilmore, T. H. (1968). British fournal of Psychiatry, 114, 93.

Hunter, R. K., Earl, C. J., and Thornicroft, S. (1964). Proceedings of the Royal Society of Medicine, 57, 758.

Hurwitz, L. J., and Montgomery, D. A. D. (1965). Archives of Neurology, 13,421 .
Hutner, S. H., Bach, M. K., and Ross, G. I. M. (1956). Fournal of Protozoology, 3, 101

Jolliffe, N., Bowman, K. M., Rosenblum, L. A., and Fein, H. D. (1940). Fournal of the American Medical Association, 114, 307.

Kalbfleisch, J. M., Lindeman, R. D., Ginn, H. E., and Smith, W. O. (1963). fournal of Clinical Investigation, 42, 1471.

Fournal of Clinical Investigation, 42, 1471.
MacIntyre, I. (1963). Scientific Basis of Medicine: Annual Reviews, p. 216. Macintyre, I. (1963). Scientific Basis of Medicint: Annual Reviews, p. 216.
Ravin, H. A. (1961). Fournal of Laboratory and Clinical Medicine, 58, 161. Ravin, H. A. (1961). Fournal of Laboratory and Clinical Medicine, 58, 161. Biochemistry, 7, 77.

Toghill, P. J., Johnston, A. W., and Smith, J. F. (1967). Fournal of Ncurology, Neurosurgery and Psychiatry, 30, 358.

Victor, M., Adams, R. D., and Cole, M. (1965). Medicine, 44, 345.

Waters, A. H., and Mollin, D. L. (1961). Fournal of Clinical Pathology, 14,

Woods, A. H., and Pendleton, L. (1925). Archives of Neurologv and Psychiatry, 13, 549.

\section{Preliminary Communications}

\section{Comparative Trial of Tetracycline, Chloramphenicol, and Trimethoprim / Sulphamethoxazole in Eradication of Vibrio cholerae El Tor}

British Medical fournal, 1970, 4, 281-282

\begin{abstract}
Summary: A comparison of tetracycline, chlor$S$ amphenicol, and trimethoprim/sulphamethoxazole showed that all hasten the eradication of Vibrio cholerae from the stools of patients with cholera.

A four-day period of tetracycline or trimethoprim/sulphamethoxazole was adequate for eradicating V. cholerae from the stools of all patients, but three days, as suggested by the W.H.O. Expert Committee, was not. Four days of chloramphenicol therapy was sufficient for most patients, but a minority required up to seven days' therapy.

Purging produced reappearance of $V$. cholerae in the stools of one-eighth of the patients who had had three successive daily negative stool cultures; such patients are a potential danger to the population.
\end{abstract}

\section{INTRODUCTION}

The efficacy of suitable antibiotics both for eradication of Vibrio cholerae from the intestinal tract and for reducing the intravenous fluid requirement has been well established (Carpenter et al., 1965; Gordon et al., 1965; Wallace et al., 1965). From the epidemiological standpoint it is vitally important that cholera patients should not leave hospital until free from $V$. cholerae. According to the recommendation made by the World Health Organization Expert Committee, administration of a suitable antibiotic in a proper dose for three consecutive days is sufficient to abolish $V$. cholerae from the stools (W.H.O., 1967). Tetracycline and chloramphenicol have been used widely and reported to be quite effective for treatment of cholera and for eradication of the organism (Carpenter et al. 1965; Kobari, 1966; Felsenfeld, 1967; W.H.O. 1967).

This study was carried out to compare the therapeutic value of a combination of trimethoprim/sulphamethoxazole with that of tetracycline and chloramphenicol, and to determine whether a three-day period of therapy is adequate to eliminate $V$. cholerae from the intestinal tract. We also studied the effect of purging on the reappearance of $V$. cholerae in the stools of patients who had had a negative stool culture on three consecutive days.

\section{METHODS}

The study was carried out in the infectious diseases ward of the Pahlavi University Hospital in Teheran during an outbreak of cholera. Stool specimens were cultured before treatment was begun and on each day of the stay in hospital. Only those patients from whose stools $V$. cholerae had been cultured were taken into the trial.

Therapy.-The patients were divided randomly into four groups, who received one of the following treatment regimens: chloramphenicol, tetracycline, trimethoprim/sulphamethoxazole, or a placebo (dextrose). A dose of 40 and 50 $\mathrm{mg} / \mathrm{kg}$. body weight to a maximum of $2 \mathrm{~g}$./day was given for tetracycline and chloramphenicol respectively in four equally divided doses. Trimethoprim/sulphamethoxazole was given in a dose of $10 \mathrm{mg}$. of trimethoprim and $50 \mathrm{mg}$. of sulphamethoxazole per $\mathrm{kg}$. of body weight to a daily maximum of $390 \mathrm{mg}$. of trimethoprim and $1.6 \mathrm{~g}$. of sulphamethoxazole ( 4 tablets) in two equal doses. Dextrose tablets were given to the placebo group twice a day. All the medications were given orally and were well tolerated. Each drug, or dextrose, was given for a minimum of three days or until a negative stool culture was obtained. After three consecutive daily stool cultures had proved negative magnesium sulphate was given as a purge in doses of $10 \mathrm{~g}$. for children and $20 \mathrm{~g}$. for adults. If the stool culture remained negative after the purge the patients were discharged. Those patients with previously negative stools who showed a positive culture after the purge were given three more days of antibiotic therapy and then discharged.

Bacteriological Studies.-Stool specimens were placed

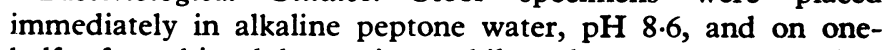
half of a thiosulphate citrate bile salt sucrose agar plate (T.C.B.S.) (Kobayashi, et al., 1963). They were incubated at $37^{\circ} \mathrm{C}$. for six to eight hours and then the other half of the T.C.B.S. plate was streaked from the growth obtained in liquid medium. After overnight incubation the suspicious colonies were tested with polyvalent cholera antiserum and if positive were then retested with Ogawa and Inaba typespecific antisera. If the suspicious colony failed to react with the polyvalent serum a sweep from the confluent area was similarly tested before discarding the culture as negative. When agglutination was positive the remnants of the colony were transferred on to a Kligler iron agar slant. The growth was serologically confirmed and then subjected to the following tests: the Voges-Proskauer reaction, indole test, haemagglutination of chicken erythrocytes, sensitivity to a disc containing 50 units of polymyxin B and to cholera phage group IV at normal test dilution, plate and tube haemolysis of sheep erythrocytes, the oxidase test, and sucrose, mannitol, and arabinose fermentation tests (Felsenfeld, 1967; W.H.O. 1967).

\section{RESULTS}

Bacteriology.-All the strains isolated were $V$. cholerae El Tor, type Inaba. They were insensitive to Mukerjee's phage IV, insensitive to polymyxin B, gave haemolysis on plates but none in tubes, and agglutinated chicken erythrocytes. They 